\title{
Enveloping Lie Algebras of Low Dimensional Leibniz Algebras
}

\author{
Massoud Amini ${ }^{1,2}$, Isamiddin Rakhimov', Seyed Jalal Langari ${ }^{2}$ \\ ${ }^{1}$ Department of Mathematics, Tarbiat Modares University, Tehran, Iran \\ ${ }^{2}$ Institute for Mathematical Research (INSPEM) \& Department of Mathematics, Selangor Darul Ehsan, Malaysia \\ E-mail:mamini@modares.ac.ir,massoud@putra.upm.edu.my, isamiddin@science.upm.edu.rny, \\ jalal_langari@yahoo.com \\ Received April 26, 2010; revised November 9, 2010; accepted June 25, 2011
}

\begin{abstract}
We calculate the enveloping Lie algebras of Leibniz algebras of dimensions two and three. We show how these Lie algebras could be used to distinguish non-isomorphic (nilpotent) Leibniz algebras of low dimension in some cases. These results could be used to associate geometric objects (loop spaces) to low dimensional Leibniz algebras.
\end{abstract}

Keywords: Leibniz Algebra, Enveloping Lie Algebra, Nilpotent Algebra

\section{Introduction}

In this paper, we work with vector spaces (and algebras) over a field $\mathrm{F}$ of characteristic 0 , although our results can be extended in obvious way to the case of vector spaces over a field of positive characteristic (not equal 2), or even over a commutative ring with unit. By an alge$\operatorname{bra}(L, \cdot)$, we mean a vector space $L$ over $\mathrm{F}$ with a (not necessarily associative) bilinear operation $: L \times L \rightarrow L$. For $x \in L, \lambda(x): L \rightarrow L ; y \rightarrow x \cdot y$ denotes the left multiplication map. Let $\operatorname{Der}(L)$ denotes the Lie sub-algebra of $g l(L)$ consisting of the derivations on $L$. Recall that a linear map $\xi \in g l(L)$ is a derivation of $(L, \cdot)$ if and only if

$$
[\xi, \lambda(x)]=\lambda(\xi x)
$$

for all $x \in L$. Here, we work with a class of algebras in which the left multiplication map has a stronger compatibility relation with derivations. These are Leibniz algebras, introduced by J. L. Loday [1], as non-antisymmetric generalizations of Lie algebras.

Definition 1.1. A Leibniz algebra $L$ is a vector space over a field $F$ equipped with a bilinear map

$$
\cdot: L \times L \rightarrow L
$$

satisfying the Leibniz identity

$$
x \cdot(y \cdot z)=(x \cdot y) \cdot z+y \cdot(x \cdot z) \text {, for all } x, y, z \in L .
$$

Obviously, a Lie algebra is a Leibniz algebra. A Leib- niz algebra is a Lie algebra if and only if

$$
x \cdot x=0 \quad(x \in L)
$$

Also, an algebra $(L, \cdot)$ is a Leibniz algebra if and only if $\lambda(x) \subseteq \operatorname{Der}(L)$ or equivalently,

$$
\lambda:(L, \cdot) \rightarrow(\operatorname{gl}(L),[\cdot, \cdot])
$$

is a homomorphism. Thus we have a homomorphism $\lambda:(L, \cdot) \rightarrow(g l(L),[\cdot, \cdot])$ when $(L, \cdot)$ is a Leibniz algebra.

Definition 1.2. If $(L, \cdot)$ is a Leibniz algebra. We may define $L^{1}=L, L^{k}=L \cdot L^{k-1}(k>1)$. The series

$$
L^{1} \supseteq L^{2} \supseteq L^{3} \supseteq \cdots
$$

is called the descending central series of $L$. If the series terminates for some positive integer $s$, then the Leibniz algebra $L$ is said to be nilpotent.

\section{Methods}

The main tool to classify Low dimensional Leibniz algebras is to find the corresponding enveloping Lie algebra and fit them in the Beck-Kolman list of low dimensional Lie algebras. Since these Lie algebras are realized as certain quotients of the given Leibniz algebras, we first need the following fact. 
Theorem 1.1. Let $\left(L_{1}, \cdot\right)$ and $\left(L_{2}, \cdot\right)$ be Leibniz algebras. If $L_{1} \cong L_{2}$ then $L_{1} / J_{1} \cong L_{2} / J_{2}$, where $J_{i}=\left\langle x \cdot x ; x \in L_{i}\right\rangle$ is the ideal generated by squares in $L_{i}$, for $i=1,2$.

Proof. Let $\phi: L_{1} \rightarrow L_{2}$ be an isomorphism. We define $\psi: L_{1} / J_{1} \rightarrow L_{2} / J_{2}$ such that $\psi\left(x+J_{1}\right)=\phi(x)+J_{2}$. It is easy to show that $\psi$ is well defined and onto, and

$$
\begin{aligned}
& \psi\left(\left(x_{1}+J_{1}\right) \cdot\left(x_{2}+J_{2}\right)\right) \\
& =\psi\left(x_{1} \cdot x_{2}+J_{1}\right)=\phi\left(x_{1} \cdot x_{2}\right)+J_{2} \\
& =\phi\left(x_{1}\right) \cdot \phi\left(x_{2}\right)+J_{2}=\phi\left(x_{1}+J_{2}\right) \cdot \phi\left(x_{2}+J_{2}\right) \\
& =\psi\left(x_{1}+J_{1}\right) \cdot \psi\left(x_{2}+J_{2}\right)
\end{aligned}
$$

Also,

$$
\begin{aligned}
& \operatorname{Ker}(\psi)=\left\{x+J_{1} ; \phi(x)+J_{2}=0\right\} \\
& =\left\{x+J_{1} ; \phi(x) \in J_{2}=\phi\left(J_{1}\right)\right\} \\
& =\left\{x+J_{1} ; x \in J_{1}\right\}=0
\end{aligned}
$$

This theorem could be used to prove that some (nilpotent) Leibniz algebras are non-isomorphic. This is important, as the nilpotent low dimensional Lie algebras are already classified [2].

Example 1.1. Let $L_{1}: e_{1} \cdot e_{1}=e_{2}, e_{2} \cdot e_{1}=e_{3}$, and $L_{2}: e_{1} \cdot e_{1}=e_{3}, e_{1} \cdot e_{2}=e_{3}, e_{2} \cdot e_{1}=e_{3}$.

Then $L_{1} / J_{1}$ is a one-dimensional abelian Lie algebra, but $L_{2} / J_{2}$ is a two-dimensional abelian Lie algebra, therefore $L_{1} / J_{1}$ is not isomorphic to $L_{2} / J_{2}$. By the previous theorem, $L_{1}$ is not isomorphic to $L_{2}$.

We noted that Leibniz algebras are non-antisymmetric in general. Hence, it is natural to consider the skewsymmetrization of a Leibniz algebra $(L, \cdot)$. This is done through the skew-symmetrized binary operation

$$
[[\cdot, \cdot]]=\frac{1}{2}(x \cdot y-y \cdot x)
$$

for $x, y \in L$. Note that, in general, $(L,[[\cdot, \cdot]])$ is not a Lie algebra. On the other hand, by definition of Leibniz algebra, $\lambda(x) \in \operatorname{Der}(L,[[, \cdot]])$ for all $x \in L$, and $\lambda:(L,[[\cdot, \cdot]) \rightarrow \operatorname{Der}(L)$ is a homomorphism of anticommutative algebras. Let

$$
J=\langle x \cdot x ; x \in L\rangle
$$

be the two-sided ideal of $(L, \cdot)$ generated by all squares. Then $J$ contains all symmetric products $x \cdot y-y \cdot x$, for $x, y \in L$, and since $\lambda(x \cdot x)=[\lambda(x), \lambda(x)]$ for all $x \in L$, we have

$$
J \subseteq \operatorname{ker}(\lambda)
$$

Let $M \subseteq L$ be any ideal containing $J$, then since $x \cdot y+M=-y \cdot x+(x \cdot y+y \cdot x)=-y \cdot x+M$ for $x, y \in L$, the Leibniz product in $L$ lifts to a Lie bracket $[\cdot, \cdot]$ in $L / M$. Conversely, if $J \subseteq M \subseteq L$ is an ideal, then the quotient $h=L / M$ is a Lie algebra. In particular, $J$ is the smallest two-sided ideal of $L$ such that $L / J$ is a Lie algebra.

Let $(h,[\cdot, \cdot])$ be a Lie, and $(L, \cdot)$ be a Leibniz algebra, we define a binary operation on the semidirect product $h \times L$ by

$$
\left[\left(\bar{e}_{i}, x\right),\left(\bar{e}_{j}, y\right)\right]=\left(\left[\bar{e}_{i}, \bar{e}_{j}\right], e_{i} \cdot y-e_{j} \cdot x\right)
$$

for $\bar{e}_{i}, \bar{e}_{i} \in h$, and $x, y \in L$, where $\bar{e}_{i}=e_{i}+M \quad$ [3]. Since $M$ contains all squares, it is clear that this operation is well defined.

Proposition 1.1. If $L$ is a nilpotent Leibniz algebra, and $M$ is an ideal of $L$ such that $J \subseteq M \subseteq \operatorname{ker}(\lambda)$ and $h=L / M$, then

1) $h$ is nilpotent Lie algebra.

2) $N=h \times L$ is a nilpotent Lie algebra.

Proof. 1) $L$ is a nilpotent Leibniz algebra, then there exist $n \in N$ such that $L \supseteq L^{2} \supseteq L^{3} \supseteq \cdots \supseteq L^{n}=\{0\}$ Therefore $L / M \supseteq L^{2} / M \supseteq L^{3} / M \supseteq \cdots \supseteq L^{n} / M=\{0\}$ Then $h \supseteq h^{2} \supseteq h^{3} \supseteq \cdots \supseteq h^{n}=\{0\}$ Thus $h$ is a nilpotent Lie algebra.

2) Clearly if $h$ and $L$ are nilpotent, then $N$ is a nilpotent Lie algebra.

The above proposition associates two Lie algebras $h$ and $N$ to a Leibniz algebra $L$. Here $h$ is a quotient of $L$, whereas $N$ is its extension. The corresponding Lie groups could be employed to associate a geometric object to $L$ [3]. We would consider the problem of classification of these geometric objects (loop spaces) in a forthcoming paper.

\section{Results}

Next let us remind the classification results for Leibniz algebras of dimension two and three [4]. We use the convention to denote the $j^{\text {th }}$ algebra of dimension $i$ by $L_{i, j}$.

Theorem 1.2. In dimension two, there are two non-isomorphic nilpotent Leibniz algebras, where $L_{2,1}$ is abelian, and $L_{2,2}$ is given by the table $e_{1} \cdot e_{1}=e_{2}$.

Theorem 1.3. In dimension three, there are five concrete and one parametric family of pairwise non isomorphic algebras.

$$
\begin{aligned}
& L_{3,1}: \text { Abelian, } \\
& L_{3,2}: e_{1} \cdot e_{1}=e_{2}, \\
& L_{3,3}: e_{1} \cdot e_{2}=e_{3}, e_{2} \cdot e_{1}=-e_{3},
\end{aligned}
$$




$$
\begin{aligned}
& L_{3,4}: e_{1} \cdot e_{1}=e_{3}, e_{2} \cdot e_{2}=\alpha e_{3}, e_{1} \cdot e_{2}=e_{3}(\alpha \in C), \\
& L_{3,5}: e_{1} \cdot e_{1}=e_{3}, e_{1} \cdot e_{2}=e_{3}, e_{2} \cdot e_{1}=e_{3}, \\
& L_{3,6}: e_{1} \cdot e_{1}=e_{2}, e_{2} \cdot e_{1}=e_{3} .
\end{aligned}
$$

\section{Discussion}

In this section, we classify the enveloping Lie algebras of Leibniz algebras of dimension two and three. This is not a trivial task, as in each case we have to identify the resulting Lie algebra as one of the known low dimensional Lie algebras [2], by carefully defining the appropriate change of basis.

In dimension two, we have $L_{2,2}: e_{1} \cdot e_{1}=e_{2}$. Then

$$
J_{2,2}:=\left\{\lambda e_{2}: \lambda \in F\right\}
$$

and

$$
\begin{aligned}
h_{2,2} & =L_{2,2} / J_{2,2}=\operatorname{span}\left\{e_{1}+J_{2,2}, e_{1} \in L_{2,2}\right\} \\
& =\operatorname{span}\left\{\bar{e}_{1}, e_{1} \in L_{2,2}\right\}
\end{aligned}
$$

Therefore $h_{2,2}$ is 1-dimensional abelian Lie algebra. Now, we consider $N_{2,2}=h_{2,2} \times L_{2,2}$ with basis elements $E_{1}=\left(\bar{e}_{1}, 0\right), E_{2}=\left(0, e_{1}\right), E_{3}=\left(0, e_{3}\right)$. The multiplication table of $N_{2,2}$ is given by

\begin{tabular}{l|lll}
{$[\cdot, \cdot]$} & $E_{1}$ & $E_{2}$ & $E_{3}$ \\
\hline$E_{1}$ & 0 & $E_{3}$ & 0 \\
$E_{2}$ & $-E_{3}$ & 0 & 0 \\
$E_{3}$ & 0 & 0 & 0
\end{tabular}

For example

$$
\begin{aligned}
{\left[E_{1}, E_{1}\right] } & =\left[\left(e_{1}+J_{2,2}, 0\right),\left(e_{1}+J_{2,2}, 0\right)\right] \\
& =\left(e_{1} \cdot e_{1}+J_{2,2}, 0\right)=\left(e_{2}+J_{2,2}, 0\right) \\
& =0
\end{aligned}
$$

and

$$
\begin{aligned}
{\left[E_{1}, E_{2}\right] } & =\left[\left(e_{1}+J_{2,2}, 0\right),\left(0, e_{1}\right)\right] \\
& =\left(0, e_{1} \cdot e_{1}\right)=\left(0, e_{2}\right)=E_{2}
\end{aligned}
$$

Thus, $N_{2,2}$ is a 3-dimensional Lie algebra with $\left[E_{1}, E_{2}\right]=E_{3}$. Briefly, we have Table 1 for dimension 2 (where the last column identifies the Lie algebra in the Beck-Kolman list [2]).

In dimension three, for each $L_{3 . k}, k=2,3,4,5,6$ we want to obtain corresponding Lie algebra $N_{3 . k}$. For $L_{3.2}$, one can show that $h_{2}$, is 2-dimensional abelian Lie algebra and $N_{3.2}$ is given by $\left[E_{1}, E_{3}\right]=E_{4}$. For $L_{3.3}$, one can prove that $J_{3,3}=\{0\}$, therefore $h_{3,3}$ is given by
Table 1. The enveloping Lie algebras of Leibniz algebras of dimension two.

\begin{tabular}{cccc}
\hline$L_{i, j}$ & \multicolumn{1}{c}{$h_{i, j}$} & $N_{i, j}=h_{i, j} \hat{A} L_{i, j}$ & $N_{i, j}$ \\
\hline$L_{2,2}$ & $\begin{array}{l}\text { 1-dimensional abelian } \\
\text { Lie algebra }\end{array}$ & {$\left[E_{1}, E_{2}\right]=E_{3}$} & $g_{3}$ \\
\hline
\end{tabular}

$$
\left[e_{1}, e_{2}\right]=e_{3},\left[e_{2}, e_{1}\right]=-e_{3} \text {, }
$$

and $N_{3,3}$ by

$$
\left[E_{1}, E_{2}\right]=E_{3}, \quad\left[E_{1}, E_{5}\right]=E_{6}, \quad\left[E_{2}, E_{4}\right]=-E_{6}
$$

Finally, for $L_{3,4}$, one can show that $L_{3,4}$ is a 2-dimensional abelian Lie algebra and $N_{3,4}$ is given by $\left[E_{1}, E_{3}\right]=E_{5},\left[E_{1}, E_{4}\right]=E_{5},\left[E_{2}, E_{4}\right]=\alpha E_{5}(\alpha \in C)$. Theref ore for 3-dimensional Leibniz algebras, we get the Table 2.

Note that in rows three and four, the enveloping Lie algebras are isomorphic, while the original Leibniz algebras are not isomorphic. The isomorphism

$N_{3,2} \cong g_{3} \oplus C^{2}$ is given by

$e_{1} \mapsto E_{1}, e_{2} \mapsto E_{3}, e_{3} \mapsto E_{4}, e_{4} \mapsto E_{2}, e_{5} \mapsto E_{5}$,

\begin{tabular}{|c|c|c|c|}
\hline$L_{i, j}$ & $h_{i, j}$ & $N_{i, j}=h_{i, j} \hat{A}{ }_{i, j}$ & $N_{i, j}$ \\
\hline$L_{3,2}$ & $\begin{array}{l}\text { 2-dimensional } \\
\text { abelian Lie } \\
\text { algebra }\end{array}$ & {$\left[E_{1}, E_{3}\right]=E_{4}$} & $g_{3} \oplus C^{2}$ \\
\hline$L_{3,3}$ & $\begin{array}{l}{\left[e_{1}, e_{2}\right]=e_{3},} \\
{\left[e_{2}, e_{1}\right]=-e_{3}}\end{array}$ & $\begin{array}{l}{\left[E_{1}, E_{2}\right]=E_{3},\left[E_{1}, E_{5}\right]=E_{6},} \\
{\left[E_{2}, E_{4}\right]=-E_{6}}\end{array}$ & $g_{6,21}$ \\
\hline$L_{3,4}$ & $\begin{array}{l}\text { 2-dimensional } \\
\text { abelian Lie } \\
\text { algebra }\end{array}$ & $\begin{array}{l}{\left[E_{1}, E_{3}\right]=E_{5},\left[E_{1}, E_{4}\right]=E_{5},} \\
{\left[E_{2}, E_{4}\right]=\alpha E_{5}(\alpha \in C) .}\end{array}$ & $g_{5,2}$ \\
\hline$L_{3,5}$ & $\begin{array}{l}\text { 2-dimensional } \\
\text { abelian Lie } \\
\text { algebra }\end{array}$ & $\begin{array}{l}{\left[E_{1}, E_{3}\right]=E_{5},\left[E_{1}, E_{4}\right]=E_{5},} \\
{\left[E_{2}, E_{3}\right]=-E_{5}}\end{array}$ & $g_{5,2}$ \\
\hline$L_{3,6}$ & $\begin{array}{l}\text { 1-dimensional } \\
\text { abelian Lie } \\
\text { algebra }\end{array}$ & {$\left[E_{1}, E_{2}\right]=E_{3}$} & $g_{3} \oplus C^{2}$ \\
\hline
\end{tabular}

$N_{3,3} \cong g_{6,21}$ by

$e_{1} \mapsto-E_{2}, e_{2} \mapsto E_{1}, e_{3} \mapsto E_{4}, e_{4} \mapsto E_{5}, e_{5} \mapsto E_{3}, e_{6} \mapsto E_{6}$

$N_{3,4} \cong g_{5,2}$ by

$e_{1} \mapsto-\alpha E_{1}, e_{2} \mapsto-E_{3}+E_{4}, e_{3} \mapsto E_{3}, e_{4} \mapsto E_{2}, e_{5} \mapsto-\alpha E_{5}$.

$N_{3,5} \cong g_{5,2}$ by

$e_{1} \mapsto-E_{1}+E_{4}, e_{2} \mapsto E_{1}, e_{3} \mapsto E_{2}, e_{4} \mapsto E_{4}, e_{5} \mapsto E_{5}$

Also, for the 2-dimensional abelian Lie algebra $L_{2,1}, J_{2,1}=\{0\}$, and $h_{2,1}$ is an abelian Lie algebra. Therefore $N_{2,1}=h_{2,1} \times L_{2,1}$ is 4-dimensional Lie algebra. Finally, for the 3-dimensional abelian Lie algebra

Table 2. The enveloping Lie algebras of Leibniz algebras of dimension three. 
$L_{3,1}, J_{3,1}=\{0\}$ and $h_{3,1}$ is an abelian Lie algebra. Therefore

$$
N_{3,1}=h_{3,1} \times L_{3,1}
$$

is a 6-dimensional Lie algebra.

\section{Conclusions}

We have classified the enveloping Lie algebras of Leibniz algebras of dimension two and three. In each case, we have identified the corresponding Lie algebra as one of the known low dimensional Lie algebras, by defining the appropriate change of basis which implements the canonical isomorphism.

There is one two dimensional Leibnitz algebra (up to isomorphism) whose corresponding Lie quotient is a 1-dimensional abelian Lie algebra. On the other hand, there are exactly five non-isomorphic three dimensional Leibnitz algebra, which correspond to three 2-dimensional abelian Lie algebras (two of which are isomorphic), one 1-dimensional abelian Lie algebra, and a 3-dimensional non-abelian Lie algebra.

\section{References}

[1] J. L. Loday, "Une version non-commutative des algebras de Lie, Les algebres de Leibniz,” Mathematics at Ecole Normale Supérieure, Vol. 39, 1993, pp. 269-293.

[2] R. E. Beck and B. Kolman, "Constructions of Nilpotent Lie Algebras over Arbitrary Fields,” In: P. S. Wang, Ed., Proceedings of 1981 ACM Symposium on Symbolic and Algebraic Computation, New York, 1981, pp. 169-174.

[3] M. K. Kinyon and A. Weinestein, "Leibniz Algebras, Courant Algebroids, and Multiplications on Homogeneous Spaces,” American Journal of Mathematics, Vol. 123, No. 3, 2001, pp. 525-550. doi:10.1353/ajm.2001.0017

[4] S. Albeverio, B. A. Omirov and I. S. Rakhimov, "Varieties of Nilpotent Complex Leibniz Algebras of Dimension Less Than Five," Communications in Algebra, Vol. 33, No. 5, 2005, pp. 1575-1585. doi:10.1081/AGB-200061038 